\title{
INVESTIGATION OF THE MICROSTRUCTURAL BANDING IN STEEL 4140
}

\author{
M. V. Maisuradze, M. A. Ryzhkov* \\ Ural Federal University, 19 Mira St., Ekaterinburg, Russian Federation \\ *Corresponding author. E-mail: mt-243@mail.ru; \\ Address for correspondence: ul. Mira, 19, 620000, Ekaterinburg, Russia.
}

The microstructural banding of the 4140 steel is studied. The dependence of the anisotropy index on the bar diameter and sampling location is established in accordance with GOST R 54570 (ASTM E 1268). A relationship between microstructural banding (i.e. anisotropy index) and toughness is shown to exist. A correlation between the kinetics of isothermal austenite transformation in the 4140 steel and the chemical inhomogeneity of the steel is discussed. formation.

Keywords: 4140 steel, microstructural banding, anisotropy index, toughness, bainite trans-

DOI: $10.17804 / 2410-9908.2017 .3 .042-052$

\section{References}

1. Eckert J.A., Howell P.R., Thompson S.W. Banding and the nature of large, irregular pearlite nodules in a hot-rolled low-alloy plate steel: a second report. Journal of Material Science, 1993, vol. 28, no. 16, pp. 4412-4420. DOI: 10.1007/BF01154950.

2. Verhoeven J.D. A review of microsegregation induced banding phenomena in steels // Journal of Materials Engineering and Performance, 2000, vol. 9, no. 3, pp. 286-291. DOI: $10.1361 / 105994900770345935$.

3. Grange R.A. Effect of microstructural banding in steel. Metallurgical Transactions, 1971, vol. 2, no. 2, pp. 417-422. DOI: 10.1007/BF02663328.

4. Caballero F. García, García-Junceda A., Capdevila C., De Andrés C.G. Evolution of microstructural banding during the manufacturing process of dual phase steels. Materials Transactions, 2006, vol. 47, no. 9, pp. 2269-2274. DOI: 10.2320/matertrans.47.2269.

5. Shi L., Yan Z., Liu Y., Yang X., Zhang Ch., Li H. Effect of acicular ferrite on banded structures in low-carbon microalloyed steel. International Journal of Minerals, Metallurgy and Materials, 2014, vol. 21, no. 12, pp. 1167-1174. DOI: 10.1007/s12613-014-1024-4.

6. Krauss G. Solidification, segregation, and banding in carbon and alloy steels. Metallurgical and Materials Transactions B, 2003, vol. 34, no. 6, pp. 781-794. DOI: 10.1007/s11663-003-0084-z.

7. Bastien P.G. The mechanism of formation of banded structures. Journal of Iron and Steel Institute, 1957, vol. 187, pp. 281-291.

8. Silin D.A., Veselov I.N., Zhukova S.Yu., Farber V.M. Microstructure and distribution of chemical elements in continuous-cast pipe blank. Steel in Translation, 2006, vol. 36, no. 4, pp. 86-90.

9. Kirkaldy J.S., Von Destinon-Forstmann J., Brigham R.J. Simulation of banding in steels. Canadian Metallurgy Quarterly, 1962, vol. 1, iss. 1, pp. 59-81. DOI: 10.1179/cmq.1962.1.1.59.

10. Morales-Rivas L., Roelofs H., Hasler S., Garcia-Mateo C., Caballero F.G. Detailed characterization of complex banding in air-cooled bainitic steels. Journal of Minerals and Metallurgy B, 2015, vol. 51, iss. 1, pp. 25-32. DOI: 10.2298/JMMB140331008M.

11. Sakir Bor A. Effect of Pearlite Banding on Mechanical Properties of Hot-rolled Steel Plates. ISIJ International, 1991, vol. 31, no. 12, pp. 1445-1446. DOI: 10.2355/isijinternational.31.1445.

12. Gulyaev A.P. Metallovedenie [Metal Science]. M., Metallurgiya Publ., 1977, 650 p. (In Russian). 
13. Shanmugam P., Pathak S.D. Some studies on the impact behavior of banded microalloyed steel. Engineering and Fracture Mechanics, 1996, vol. 53, iss. 5, pp. 991-1005. DOI: 10.1016/0013-7944(95)00159-X.

14. Caballero F.G., Chao J., Cornide J., García-Mateo C., Santofimia M.J., Capdevila C. Toughness deterioration in advanced high strength bainitic steels. Materials Science and Engineering A, 2009, vol. 525, pp. 87-95. DOI: 10.1016/j.msea.2009.06.034.

15. ASTM E 1268-01. Standard Practice for Assessing the Degree of Banding or Orientation of Microstructures, ASTM, 2001, 29 p.

16. D'Errico F. Failures induced by abnormal banding in steels. Journal of Failure Analysis and Prevention, 2010, vol. 10, iss. 5, pp. 351-360. DOI: 10.1007/s11668-010-9374-3.

17. Rodionova I.G., Zaitsev A.I., Baklanova O.N. Effect of Carbon Steel Structural Inhomogeneity on Corrosion Resistance in Chlorine-Containing Media. Metallurgist, 2016, vol. 59, nos. 9-10, pp. 774-783. DOI: 10.1007/s11015-016-0173-2.

18. Rivera-Diaz-del-Castillo P.E.J., Van der Zwaag S. Assuring Microstructural Homogeneity in Dual Phase and TRIP Steels. Steel Research International, 2004, vol. 75, no. 11, pp. 711-715. DOI: $10.1002 /$ srin.200405832.

19. Xu W., Rivera-Diaz-del-Castillo P.E.J., Van der Zwaag S. Ferrite/Pearlite Band Prevention in Dual Phase and TRIP Steels: Model Development. ISIJ International, 2005, vol. 45, no. 3, pp. 380-387. DOI: 10.2355/isijinternational.45.380.

20. Marder A.R. Deformation characteristics of dual-phase steels. Metallurgical Transactions A, 1982, vol. 13, pp. 85-92. DOI: 10.1007/BF02642418.

21. Cai X.L., Garratt-Reed A.J., Owen W.S. The development of some dual-phase steel structures from different starting microstructures. Metallurgical Transactions A, 1985, vol. 16, iss. 4, pp. 543-557. DOI: 10.1007/BF02814228.

22. Jatczak C.F., Girardi D.J., Rowland E.S. On banding in steel. Transactions of ASM, 1956, vol. 48, pp. 279-305.

23. GOST 5640-68. Metallographic method for determination of microstructure of sheets and bands. M., Izdatelstvo Standartov Publ., 1988, 18 p. (In Russian).

24. GOST 801-78. Bearing steel. Specifications. M., Izdatelstvo Standartov Publ., 1979, 45 p. (In Russian).

25. UNI 8449. Classification of banded structure in case hardening steels, UNI, 1983, $15 \mathrm{p}$.

26. Tasan C.C., Hoefnagels J.P.M., Geers M.G.D. Microstructural banding effects clarified through micrographic digital image correlation. Scripta Materialia, 2010, vol. 62, iss. 11, pp. 835-838. DOI: $10.1016 /$ j.scriptamat.2010.02.014.

27. Krebs B., Hazotte A., Germain L. Quantitative analysis of banded structures in dual-phase steels. Image Analysis and Stereology, 2010, vol. 29, no. 2, pp. 85-90. DOI: 10.5566/ias.v29. p. 85-90.

28. GOST $R$ 54570-2011. Assessing the degree of banding or orientation of microstructures. M., Standartinform Publ., 2012, 36 p. (In Russian). 
Подана в журнал: 03.07.2017

УДК 669.017

DOI: $10.17804 / 2410-9908.2017 .3 .042-052$

\title{
ИССЛЕДОВАНИЕ СТРУКТУРНОЙ ПОЛОСЧАТОСТИ В КОНСТРУКЦИОННОЙ СТАЛИ 40ХН2МА
}

\author{
М. В. Майсурадзе, М. А. Рыжков \\ ФГАОУ ВО УрФУ, ул. Мира, 19, г. Екатеринбург, Российская Федеращия \\ *Ответственный автор. Электронная почта: mt-243@mail.ru; \\ адрес для переписки: 620000, г. Екатеринбург, ул. Мира, 19.
}

Проведено количественное определение степени структурной полосчатости в конструкционной легированной стали 40ХН2МА. Установлена зависимость индекса анизотропии, определенного согласно ГОСТ Р 54570 (ASTM E 1268), от диаметра проката и места отбора пробы. Показано, что существует зависимость между структурной полосчатостью и анизотропией ударной вязкости. Рассмотрена взаимосвязь между кинетикой изотермического превращения аустенита в стали 40ХН2МА и химической неоднородностью стали, являющейся причиной полосчатости микроструктуры.

Ключевые слова: сталь 40ХН2МА, структурная полосчатость, индекс анизотропии, ударная вязкость, бейнитное превращение.

\section{1. Введение}

Структурная полосчатость характерна для горячекатаной углеродистой и легированной стали [1...5]. Ее происхождение обусловлено химической ликвацией легирующих элементов и примесей еще на стадии кристаллизации стали, когда происходит образование дендритных кристаллов. Междендритные области обычно имеют повышенное содержание таких легирующих элементов, как $\mathrm{Mn}, \mathrm{Cr}, \mathrm{Si}, \mathrm{Mo} \mathrm{и} \mathrm{др.} \mathrm{[6-10].} \mathrm{Горячая} \mathrm{про-}$ катка или ковка слитка приводит к ориентированию дендритной структуры вдоль направления деформации, а в результате последующей термической обработки формируется структурная полосчатость, т. е. чередующиеся полосы, содержащие различные фазы и структурные составляющие - полосы феррита и перлита, полосы мартенсита в ферритной или бейнитной матрице и др. [7-15].

Структурная полосчатость является причиной анизотропии механических свойств стали, поскольку вдоль полос облегчено распространение трещин [16]. Кроме того, структурная полосчатость негативно сказывается на коррозионной стойкости сталей [17]. Многочисленные работы, посвященные проблеме структурной полосчатости, позволили понять механизм формирования полос. Однако предлагаемые технологические решения, позволяющие уменьшить отрицательное влияние структурной полосчатости $[4,18,19]$ или полностью устранить причину ее образования [20,21], не всегда целесообразны [3], а в ряде случаев технологически не реализуемы [22].

В настоящее время существует ряд стандартов, позволяющих оценить степень структурной полосчатости в сталях, однако большинство из них основано на сравнении наблюдаемой микроструктуры стали после термической обработки с эталонными шкалами [23-25], что приводит к неточной качественной оценке.

Количественная оценка структурной полосчатости является ключевым фактором, позволяющим усовершенствовать существующие или разработать новые технологии производства стального проката или поковок с повышенной однородностью микро- 
структуры [15, 26-28], что весьма актуально при обеспечении надлежащего качества готовых ответственных изделий.

\section{2. Материал и методика}

В качестве исследуемого материала выбрана конструкционная легированная сталь 40ХН2МА, используемая в современном машиностроении для изготовления широкой номенклатуры ответственных деталей, таких как валы, оси, зубчатые колеса и т. п. Химический состав исследуемых плавок стали приведен в табл. 1. Сталь поставлялась в виде круглого горячекатаного проката диаметром 50-190 мм после отжига в условиях завода-изготовителя.

Таблица 1 - Химический состав исследуемой стали 40ХН2МА, мас. \%

\begin{tabular}{|c|c|c|c|c|c|c|c|c|c|c|}
\hline $\begin{array}{c}\text { № } \\
\text { плавки }\end{array}$ & Диаметр & $\mathrm{C}$ & $\mathrm{Si}$ & $\mathrm{Mn}$ & $\mathrm{Cr}$ & $\mathrm{Ni}$ & $\mathrm{Mo}$ & $\mathrm{Cu}$ & $\mathrm{S}$ & $\mathrm{P}$ \\
\hline 1 & $\begin{array}{c}110 \mathrm{Mм} \\
140 \mathrm{Mм} \\
190 \mathrm{Mм}\end{array}$ & 0,42 & 0,29 & 0,71 & 0,75 & 1,44 & 0,27 & 0,03 & 0,003 & 0,012 \\
\hline 2 & $50 \mathrm{Mм}$ & 0,40 & 0,20 & 0,54 & 0,69 & 1,27 & 0,18 & 0,22 & 0,009 & 0,011 \\
\hline
\end{tabular}

Для исследования зависимости структурной полосчатости от диаметра проката, от стали 40ХН2МА были отобраны образцы размером $10 \times 10 \times 10$ мм из трех точек поперечного сечения: поверхность, середина радиуса и центр. Температура нагрева образцов под закалку составляла $850{ }^{\circ} \mathrm{C}$, продолжительность выдержки 60 мин. Охлаждение производилось в закалочном масле И-20А с температурой $30-40{ }^{\circ} \mathrm{C}$.

Количественная оценка структурной полосчатости производилась по методике стандарта ГОСТ Р 54570 [29] (ASTM Е 1268 [15]). Анализировалось 15 полей зрения на продольных шлифах. Поля зрения ориентировались таким образом, чтобы микроструктурные полосы на изображении были направлены строго вертикально. На каждое поле зрения накладывалась тестовая сетка, состоящая из горизонтальных и вертикальных линий. После этого производился подсчет количества пересечений светлых полос микроструктуры с горизонтальными $\left(\mathrm{N}_{\text {перп }}\right)$ и вертикальными $\left(\mathrm{N}_{\text {парал }}\right)$ линиями сетки. На основании полученных данных был рассчитан индекс анизотропии $\mathrm{AI}$ как отношение $\mathrm{N}_{\text {перп }} / \mathrm{N}_{\text {парал. }}$

Для исследования процесса протекания бейнитного превращения в стали 40ХН2МА при изотермической выдержке образцы, отобранные от центральной зоны проката диаметром 50 мм (плавка № 2), подвергались термической обработке по следующему режиму: нагрев до температуры $850{ }^{\circ} \mathrm{C}$, выдержка 60 минут, перенос в печьванну с расплавом соли $\left(50 \% \mathrm{NaNo}_{3}, 50 \% \mathrm{KNO}_{3}\right)$, выдержка в расплаве соли при температуре $400{ }^{\circ} \mathrm{C}$ в течение $15 \ldots 1000$ с и окончательное охлаждение в воде для того, чтобы непревращенный аустенит претерпел мартенситное превращение.

Микроструктура исследуемых марок стали изучалась при помощи оптической микроскопии при увеличении $\times 5 \ldots \times 100$. Количественная оценка доли бейнита в структуре образца в целом и в отдельных структурных полосах производилась при помощи коммерческого программного обеспечения путем бинаризации изображения и подсчёта количества черных (соответствующих бейнитной структуре) и белых (соответствующих структуре мартенсита) пикселей в выделенных областях микрофотографии. Для каждого времени выдержки анализировалось не менее 15 полей зрения, на каждом из которых наблюдалось не менее 15-20 структурных полос с различным содержанием бейнита.

Для наглядного представления полученных результатов и оценки изменения кинетики бейнитного превращения в полосах с различной устойчивостью переохлажденного аустенита все полосы с различным содержанием бейнита были упорядочены в вариационный ряд по 
доле бейнита. Для каждой полосы была определена занимаемая ею площадь на фотографии, $F_{i}$ (в пикселях), а также рассчитана ее относительная площадь, $F_{i}{ }^{\prime}$, по формуле:

$$
F_{i}{ }^{\prime}=F_{i} / F_{0},
$$

где $F_{0}$ - суммарная исследованная площадь образца (в пикселях).

Впоследствии вариационный ряд, полученный для каждого времени выдержки, был разделен на 10 интервалов по относительной площади, занимаемой полосами. Таким образом, каждый из интервалов охватывал 10 \% исследуемой площади образца. Для каждого интервала была определена средняя доля бейнита попавших в него полос.

Микрорентгеноспектральный анализ полос с различной устойчивостью аустенита стали 40ХН2МА производился при помощи электронного микроскопа Jeol JSM 6490, оснащенного приставкой для комбинированного энергодисперсионного и волнового микроанализа Oxford Inca Energy 350.

\section{3. Результаты и обсуждение}

\section{1. Влияние диаметра проката на структурную полосчатость}

Исследовано изменение структурной полосчатости в разных зонах поперечного сечения проката в зависимости от его диаметра. Для этого от проката диаметром 110 мм, 140 мм и 190 мм, полученного из стали одной плавки, были отобраны образцы из трех зон поперечного сечения - поверхность, середина радиуса и центр. Металлографическое исследование структурной полосчатости производилось на образцах, закаленных в масле. На рис. 1 представлена микроструктура поверхностной и центральной зон проката из стали 40ХН2МА диаметром 110 и 190 мм. Наблюдаются чередующиеся полосы мартенситной структуры разной травимости.

Микрорентгеноспектральный анализ, проведенный для полос центральной зоны проката диаметром 190 мм, показал, что в темных полосах микроструктуры содержание таких элементов, как $\mathrm{Cr}, \mathrm{Ni}$, Мо и $\mathrm{Mn}$, ниже, чем в среднем по образцу (табл. 2). Это подтверждает ликвационную природу структурных полос [6, 12]. Различный уровень легирования отдельных полос может приводить к различию в устойчивости аустенита и формированию неоднородной микроструктуры при непрерывном охлаждении от температуры аустенитизации, изотермической обработке или отпуске мартенситной структуры.

Установлено, что поверхностная зона проката обладает более однородной структурой: полосы более узкие, с диффузными границами. Рассчитанный индекс анизотропии для поверхностной зоны проката диаметром 110 мм составил 2,6, а для проката диаметром 190 мм - 1,6. Для центральной зоны проката характерна более выраженная структурная полосчатость. При этом полосы в центральной зоне проката диаметром 110 мм имеют более нелинейную, изогнутую форму по сравнению с прокатом диаметром 190 мм. Это связано с большей степенью деформации, полученной прутком диаметром 110 мм при горячей прокатке исходного слитка. Индекс анизотропии для центральной зоны проката диаметром 110 и 190 мм существенно различается и составляет 3,8 и 1,7 соответственно. 


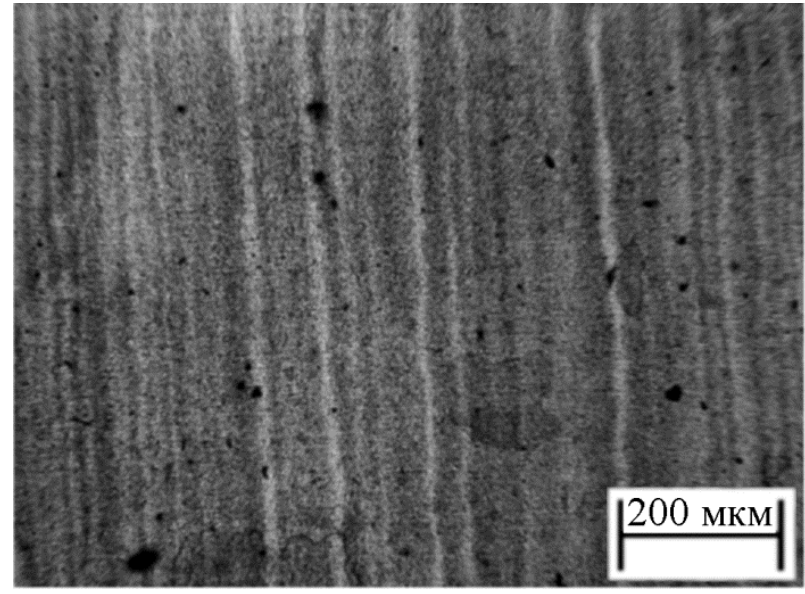

$a$

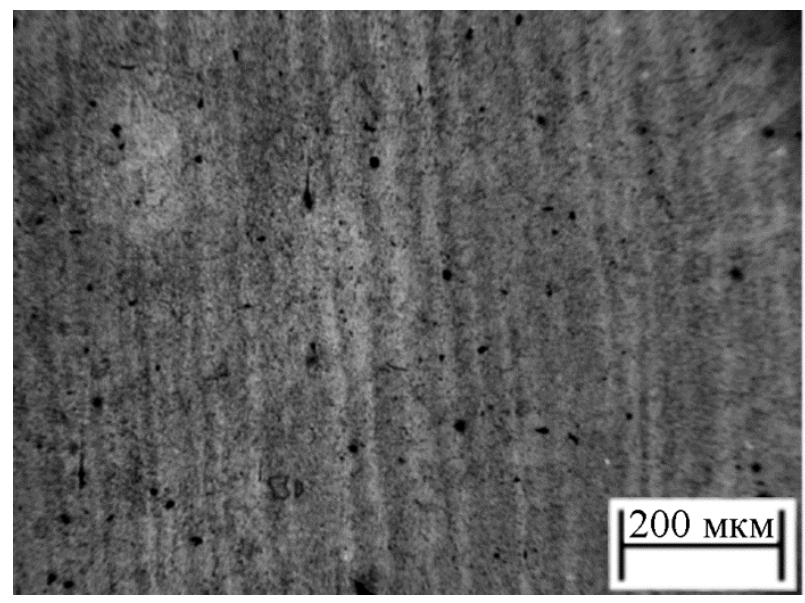

B

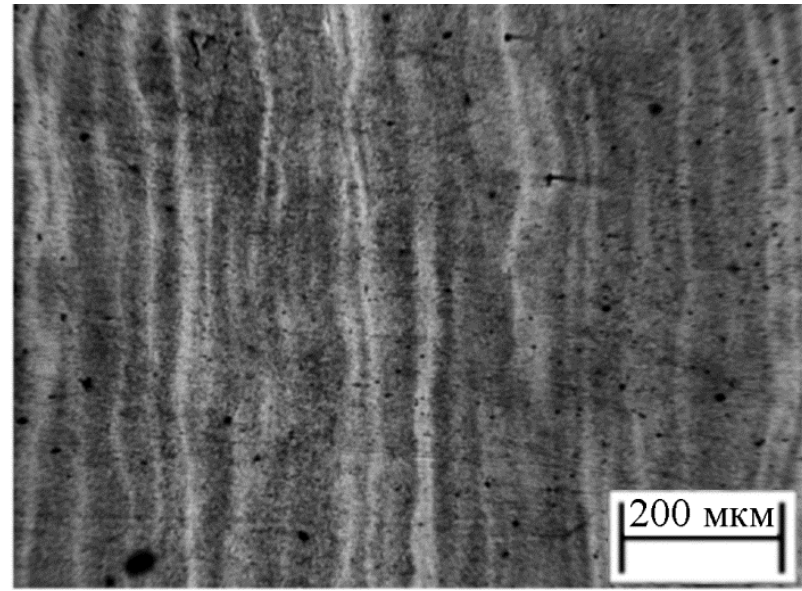

6

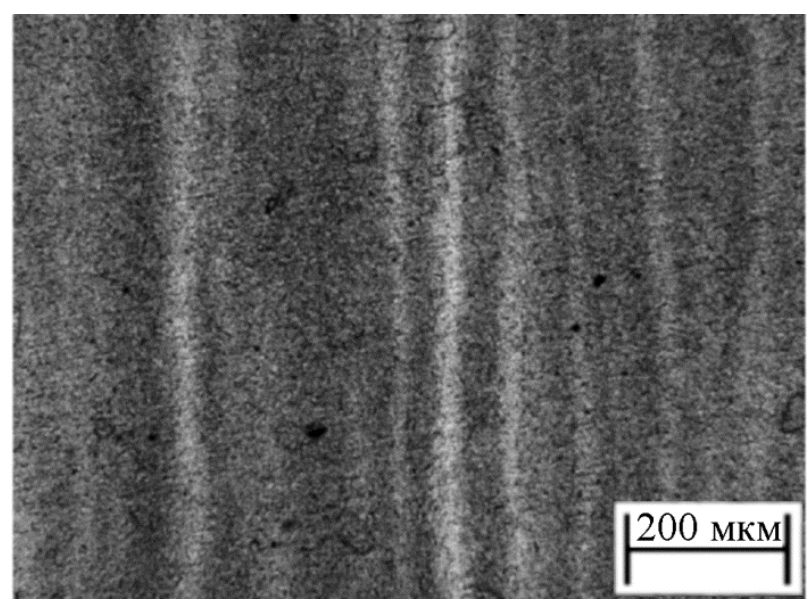

2

Рис. 1. Микроструктура поверхностной $(a, b)$ и центральной $(\sigma, 2)$ зоны проката из стали 40XН2МА после закалки в масло: $a, \sigma$ - диаметр проката 110 мм; в, г - диаметр проката 190 мм

Таблица 2 - Химический состав, мас. \%, образца в целом и темных полос микроструктуры проката диаметром 190 мм из стали 40ХН2МА (центральная зона)

\begin{tabular}{|l|c|c|c|c|c|}
\hline \multicolumn{1}{|c|}{ Анализируемая область } & $\mathrm{Si}$ & $\mathrm{Mn}$ & $\mathrm{Cr}$ & $\mathrm{Ni}$ & $\mathrm{Mo}$ \\
\hline Темные полосы & 0,29 & 0,60 & 0,71 & 1,33 & 0,19 \\
\hline Среднее содержание в образце & 0,29 & 0,73 & 0,77 & 1,45 & 0,33 \\
\hline
\end{tabular}

На основании полученных данных построена зависимость индекса анизотропии в различных точках поперечного сечения от диаметра проката (рис. 2). С уменьшением диаметра проката, а следовательно, с повышением степени деформации при горячей прокатке, индекс анизотропии стали повышается. Наименьший прирост индекса анизотропии наблюдается в поверхностной зоне проката. Это связано с тем, что поверхностная зона металла наиболее интенсивно деформируется при прокатке и химическая неоднородность, являющаяся причиной структурной полосчатости, в некоторой степени уменьшается, в отличие от глубинных слоев металла. 


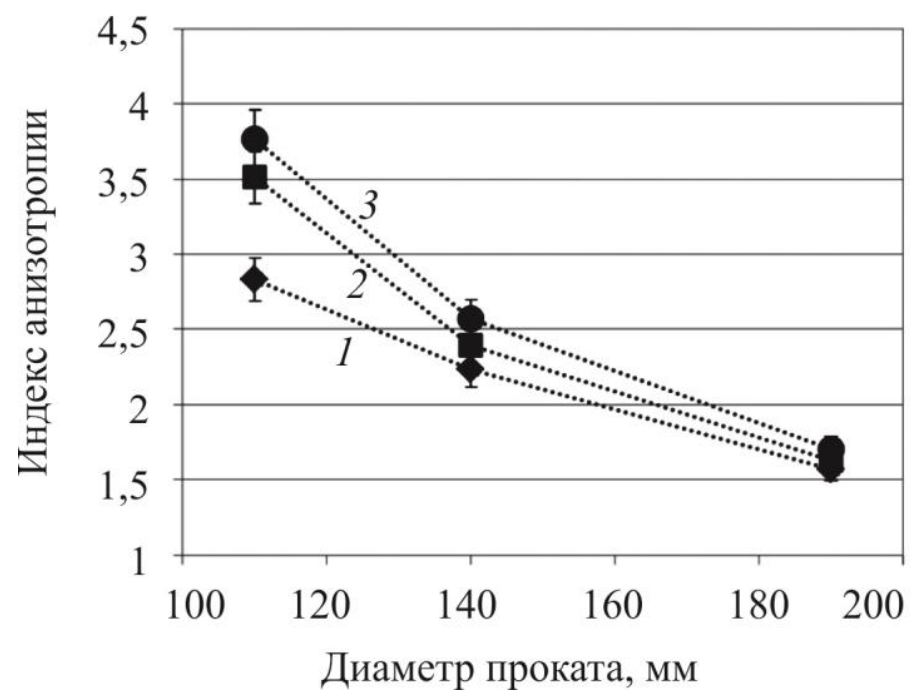

Рис. 2. График зависимости индекса анизотропии для различных зон поперечного сечения от диаметра проката из стали 40ХН2МА: 1 - поверхность; 2 - 1/2 радиуса; 3 - центр

Тенденцию к повышению неоднородности структуры с увеличением степени деформации при прокатке можно объяснить тем фактом, что скорость деформации существенно выше скорости диффузии легирующих элементов. В результате при прокатке происходит сжатие и вытягивание междендритных областей без значительного перераспределения в них легирующих элементов, что и является причиной большего количества полос и более резких границ между полосами в структуре проката меньшего диаметра.

\section{2. Влияние структурной полосчатости на формирование микроструктуры}

На рис. 3 представлена микроструктура проката диаметром 50 мм (плавка № 2) из стали 40ХН2МА после реализации изотермической выдержки при температуре $400{ }^{\circ} \mathrm{C}$ в течение различного времени выдержки. Темная составляющая микроструктуры соответствует бейниту, образовавшемуся при изотермической выдержке, светлая составляющая - мартенсит, сформировавшийся при охлаждении в воде от температуры выдержки. С увеличением времени выдержки средняя доля бейнита в исследуемой стали возрастает от 0,11 до 0,72. При этом в структуре наблюдается существенное различие в количестве образовавшегося бейнита в отдельных полосах при любой продолжительности выдержки. К примеру, в случае выдержки в течение 40 с, в отдельных полосах образуется до 0,85 бейнита, при этом также встречаются полосы, в которых количество бейнита не превышает 0,05 .

На рис. 4 представлено изменение доли бейнита в стали 40ХН2МА в зависимости от времени выдержки в интервале $15 . .60$ с. На графике представлена кинетика превращения в полосах с максимальной и минимальной устойчивостью переохлажденного аустенита. Разброс доли бейнита между отдельными полосами составляет 0,01...0,40 для времени выдержки 15 с и 0,07...0,94 для времени выдержки 60 с. То есть с увеличением времени выдержки в интервале $15 . .60$ с наблюдается увеличение разброса доли бейнита в структуре полос исследуемой стали 40ХН2МА.

Тем не менее анализ степени полосчатости микроструктуры стали 40ХН2МА по стандарту ГОСТ Р 54570 (ASTM E 1268) показал, что с увеличением времени выдержки вплоть до 1000 с (до завершения бейнитного превращения) индекс анизотропии не изменяется и составляет $1,7 \pm 0,3$. Таким образом, продолжительность выдержки не оказывает влияния на проявление структурной полосчатости в исследуемой стали, однако анализ изображений будет в значительной степени облегчен в случае формирования наиболее контрастной микроструктуры. Этому условию удовлетворяет микроструктура, сформированная в результате выдержки в течение 40 с (рис. 3 б). Микроструктура в этом случае представляет собой четко выраженные полосы бейнитной и мартенситной структуры. 


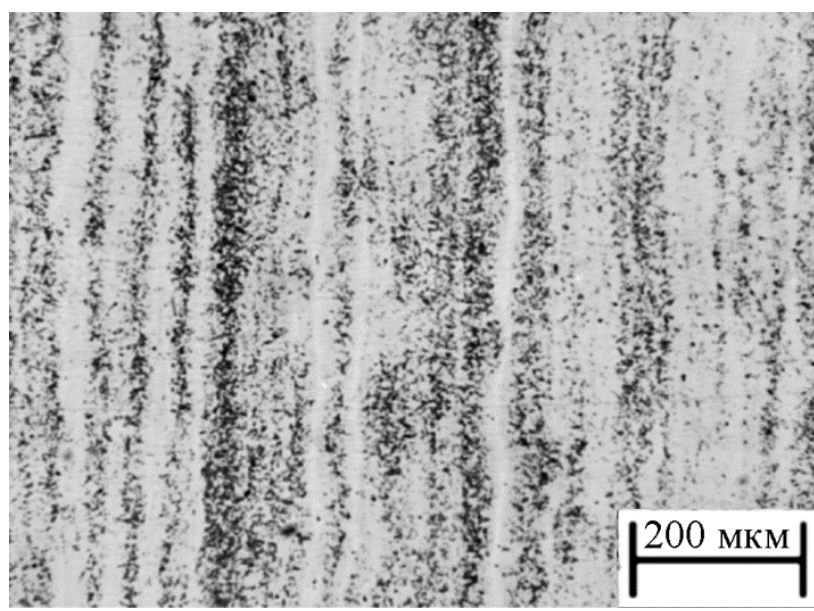

$a$

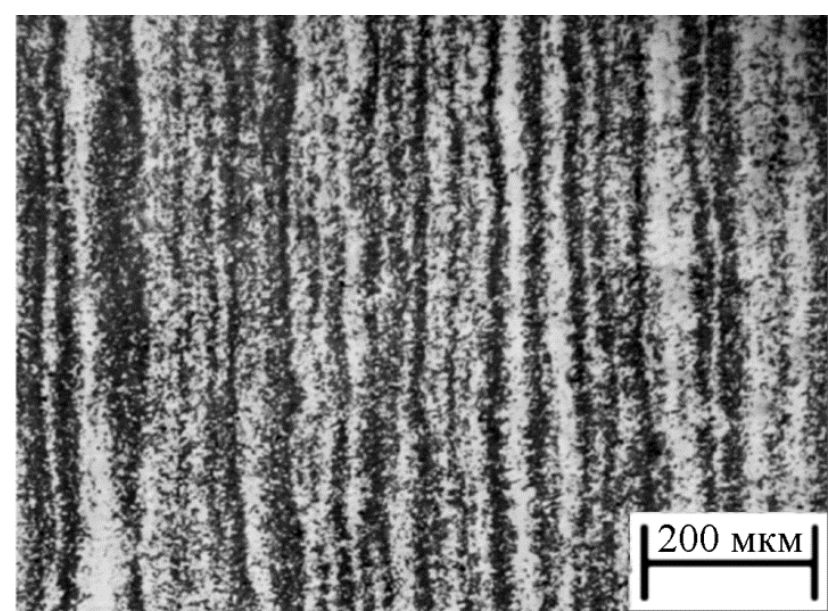

$\sigma$

Рис. 3. Микроструктура стали 40ХН2МА после изотермической выдержки при температуре $400{ }^{\circ} \mathrm{C}$ в течение 25 с (a) и 40 с (б)

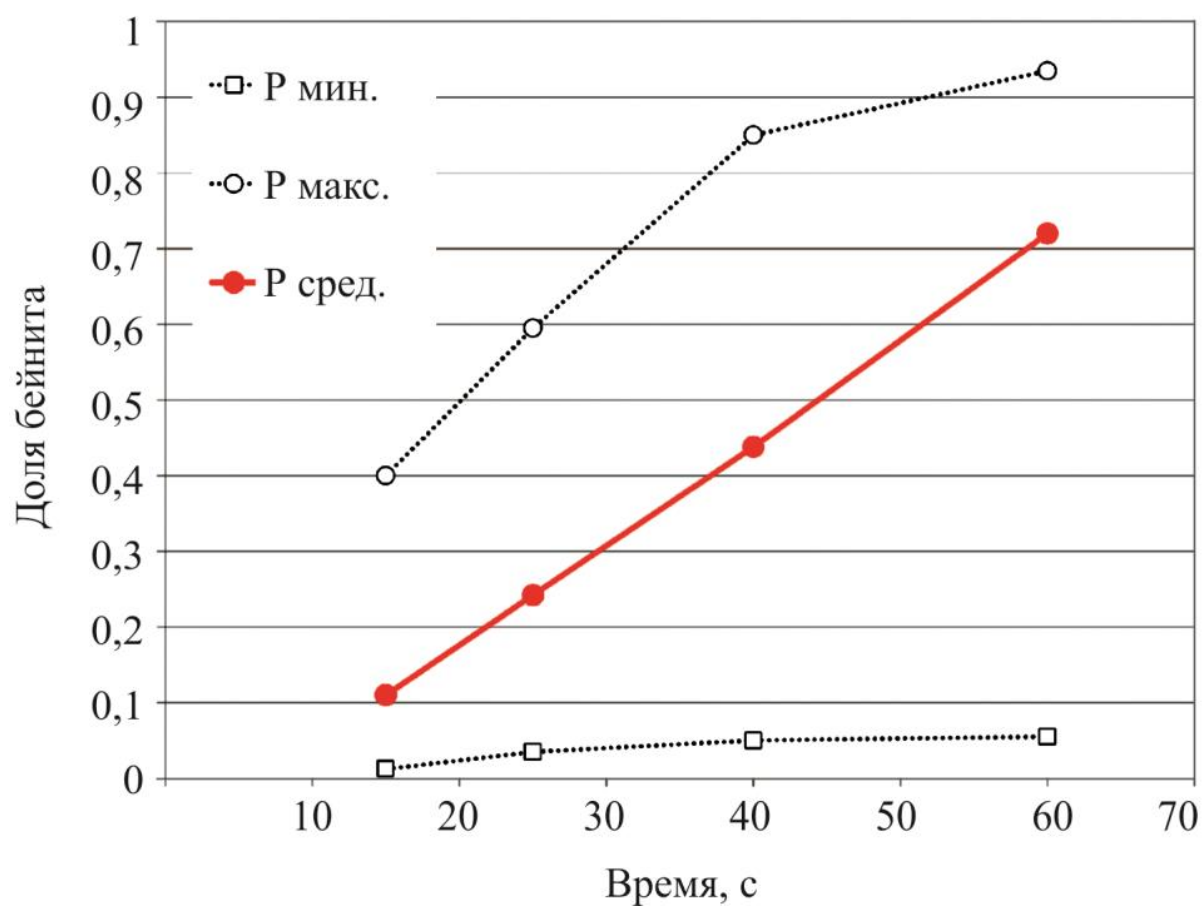

Рис. 4. Изменение доли бейнита в стали 40ХН2МА с течением времени изотермической выдержки

Статистический анализ полосчатой структуры показал, что средняя доля бейнита в стали не соответствует середине интервала разброса доли бейнита при любой продолжительности выдержки. Это связано с тем, что полосы с различным количеством бейнита занимают различную площадь на исследованной поверхности образца. На рис. 5 приведена зависимость доли бейнита от времени выдержки для десяти интервалов относительной площади, занимаемой полосами (Р1...Р10). Таким образом, каждому маркеру Р1...P10 на рис. 5 соответствует $10 \%$ от исследованной площади образца. Как видно, кинетика, соответствующая изменению средней доли бейнита в стали, наблюдается лишь для $10 \ldots 30$ \% исследованной площади образца. В остальном объеме образца превращение идет с иной скоростью. 


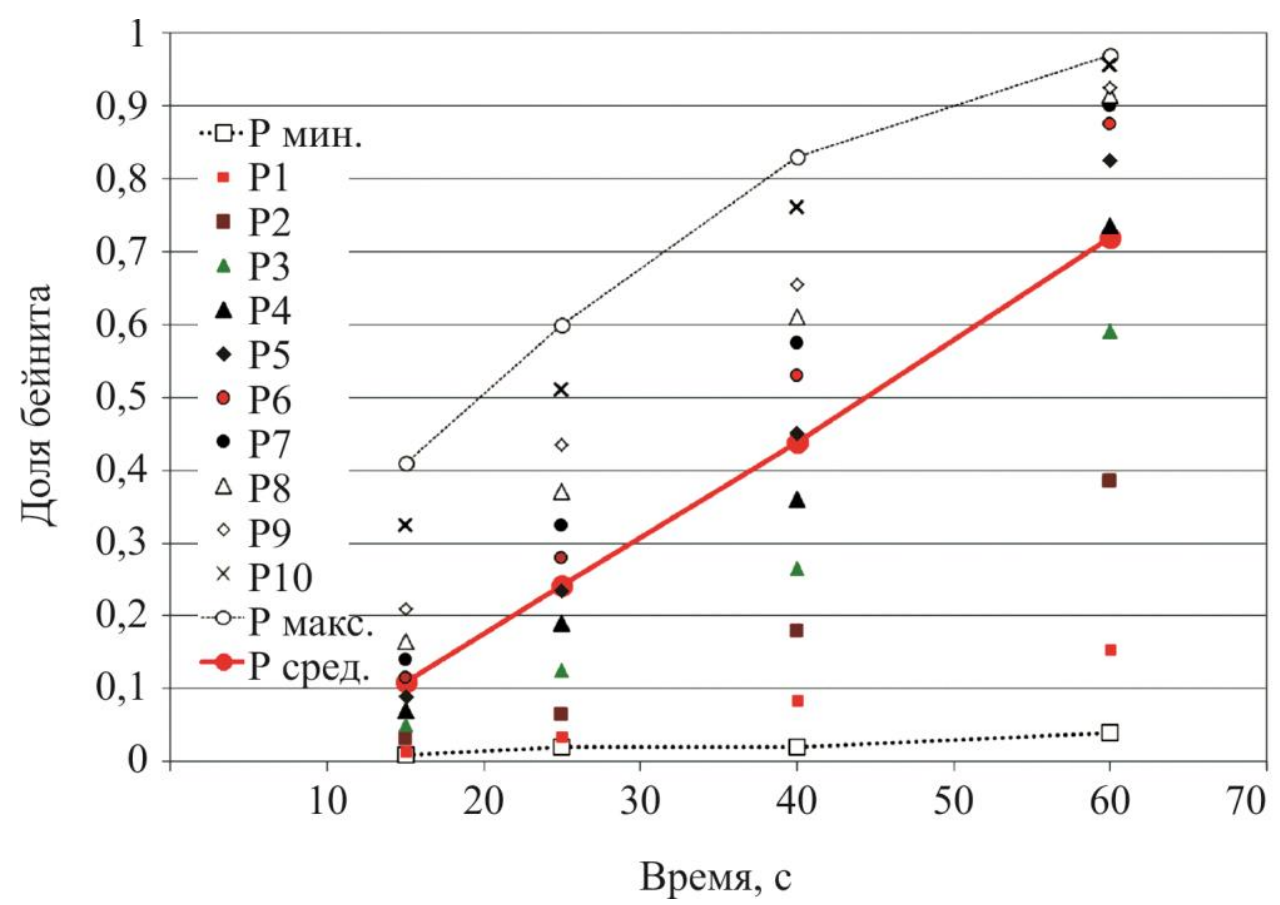

Рис. 5. Изменение доли бейнита в отдельных группах полос стали 40ХН2МА с течением времени изотермической выдержки (каждому маркеру Р 1 ...P10 соответствует 10 \% от исследованной площади образца)

Был проведен микрорентгеноспектральный анализ полос, содержащих максимальное и минимальное количество бейнита после изотермической выдержки в течение 60 с. Всего было проанализировано 20 полос. В результате установлено, что наибольший разброс имеют следующие легирующие элементы (рис. 6): $\mathrm{Mn}-0,55 \ldots 0,95$ мас. \%; $\mathrm{Cr}-0,6 \ldots 0,9$ мас. \%; $\mathrm{Ni}-1,2 \ldots 1,5$ мас. \%; Мо - 0,10...0,45 мас. \%. Согласно литературным данным [6...8], в полосах, содержащих повышенное количество марганца и молибдена, также должно содержаться повышенное количество углерода.

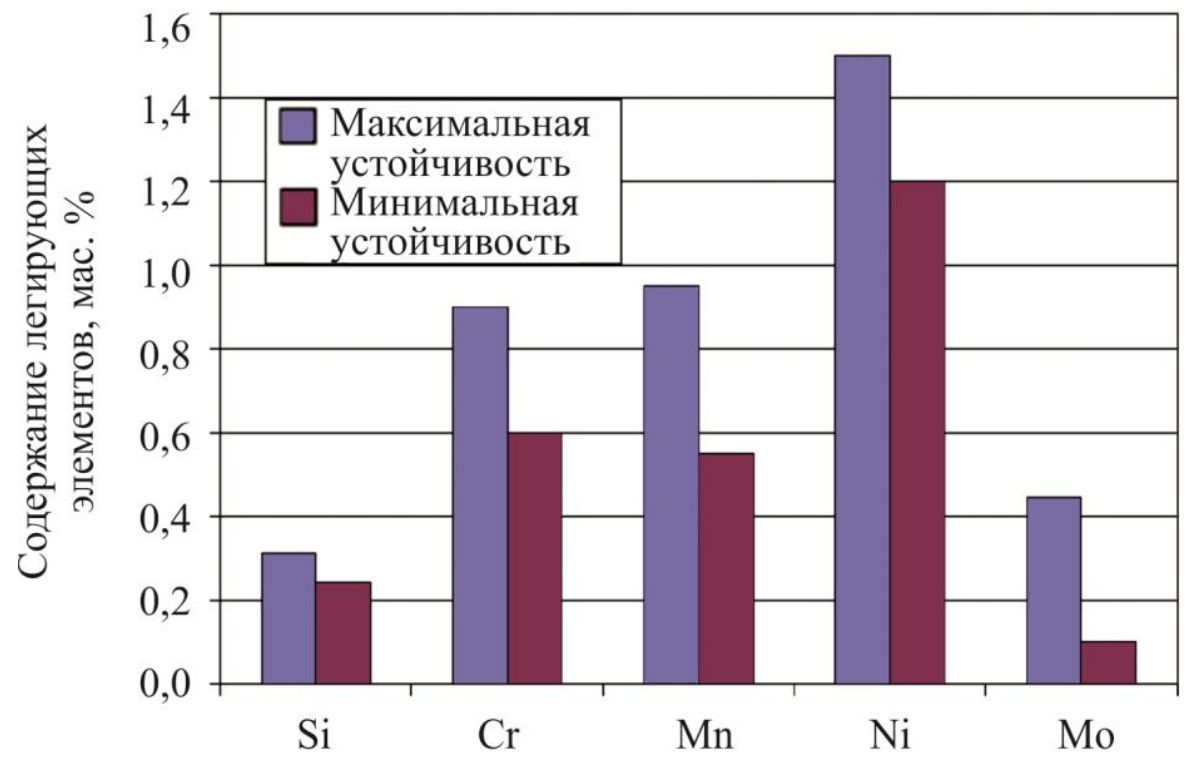

Рис. 6. Содержание легирующих элементов в различных полосах с минимальной и максимальной устойчивостью переохлажденного аустенита в стали 40ХН2MА 
Таким образом, на ширину интервала, в котором происходит изменение доли бейнита в отдельных полосах стали с течением времени, оказывает непосредственное влияние химическая неоднородность, что также было отмечено ранее в работах $[2,3,6,7]$.

Учет влияния химической неоднородности на кинетику протекания превращений в сталях может существенно повысить адекватность расчетных методов моделирования процессов термической обработки изделий.

\section{4. Заключение}

1. Проведена количественная оценка структурной полосчатости по сечению проката диаметром 110...190 мм из стали 40ХН2МА. Установлено, что с уменьшением диаметра проката индекс анизотропии возрастает для всех зон сечения проката: для поверхности от 1,6 до 2,6; для центра - от 1,7 до 3,8. Меньше всего индекс анизотропии повышается для поверхностной зоны проката, что связано с условиями деформации при горячей прокатке, вследствие чего происходит частичное выравнивание химического состава отдельных полос металла.

2. Установлено, что индекс анизотропии, определенный по методике ГОСТ Р 54570 (ASTM E 1268), не зависит от продолжительности изотермической выдержки при температуре $400{ }^{\circ} \mathrm{C}$ и составляет для центральной зоны исследуемого проката диаметром

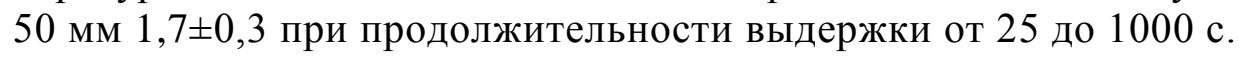

3. Установлено влияние структурной неоднородности на кинетику изотермического бейнитного превращения в стали 40ХН2МА. Показано, что разность содержания бейнита между отдельными полосами на разных этапах превращения может достигать $40 \ldots 90 \%$. Это связано с различием химического состава отдельных полос стали: разброс концентрации $\mathrm{Mn}$ составляет 0,55...0,95 мас. \%; $\mathrm{Cr}-0,6 \ldots 0,9$ мас. \%; $\mathrm{Ni}-1,2 \ldots 1,5$ мас. \%; Мо - 0,10...0,45 мас. \%.

\section{Благодарность}

Работа выполнена при финансовой поддержке постановления № 211 Правительства Российской Федераџии, контракт № 02.A03.21.0006, в рамках государственного задания Министерства образования и науки РФ, проект № 11.1465.2014/K, а также в рамках Гранта Президента РФ для молодых ученых - кандидатов наук МК-7929.2016.8.

\section{Литература}

1. Eckert J. A., Howell P. R., Thompson S. W. Banding and the nature of large, irregular pearlite nodules in a hot-rolled low-alloy plate steel: a second report // Journal of Material Science. 1993. - Vol. 28, no. 16. - P. 4412-4420. - DOI: 10.1007/BF01154950.

2. Verhoeven J. D. A review of microsegregation induced banding phenomena in steels // Journal of Materials Engineering and Performance. - 2000. - Vol. 9, no. 3. - P. 286-291. DOI: $10.1361 / 105994900770345935$.

3. Grange R. A. Effect of microstructural banding in steel // Metallurgical Transactions. 1971. - Vol. 2, no. 2. - P. 417-422. - DOI: 10.1007/BF02663328.

4. Evolution of microstructural banding during the manufacturing process of dual phase steels / F. García Caballero, A. García-Junceda, C. Capdevila, C. G. de Andrés // Materials Transactions. 2006. - Vol. 47, no. 9. - P. 2269-2274. - DOI: 10.2320/matertrans.47.2269.

5. Effect of acicular ferrite on banded structures in low-carbon microalloyed steel / L. Shi, Z. Yan, Y. Liu, X. Yang, Ch. Zhang, H. Li // International Journal of Minerals, Metallurgy and Materials. - 2014. - Vol. 21, no. 12. - P. 1167-1174. - DOI: 10.1007/s12613-014-1024-4.

6. Krauss G. Solidification, segregation, and banding in carbon and alloy steels // Metallurgical and Materials Transactions B. - 2003. - Vol. 34, no. 6. - P. 781-794. - DOI: 10.1007/s11663-003-0084-Z. 7. Bastien P. G. The mechanism of formation of banded structures // Journal of Iron and Steel Institute. - 1957. - Vol. 187. - P. 281-291. 
8. Microstructure and distribution of chemical elements in continuous-cast pipe blank / D. A. Silin, I. N. Veselov, S. Yu. Zhukova, V. M. Farber // Steel in Translation. - 2006. - Vol. 36, no. 4. - P. 86-90.

9. Kirkaldy J. S., Von Destinon-Forstmann J., Brigham R. J. Simulation of banding in steels // Canadian Metallurgy Quarterly. - 1962. - Vol. 1, iss. 1. - P. 59-81. - DOI: 10.1179/cmq.1962.1.1.59.

10. Detailed characterization of complex banding in air-cooled bainitic steels / L. MoralesRivas, H. Roelofs, S. Hasler, C. Garcia-Mateo, F. G. Caballero // Journal of Minerals and Metallurgy B. - 2015. - Vol. 51, no. 1. - P. 25-32. - DOI: 10.2298/JMMB140331008M.

11. Sakir Bor A. Effect of Pearlite Banding on Mechanical Properties of Hot-rolled Steel Plates // ISIJ International. - 1991. - Vol. 31, no. 12 - P. 1445-1446. - DOI: 10.2355/isijinternational.31.1445.

12. Гуляев А. П. Металловедение. - М. : Металлургия, 1977. - 650 с.

13. Shanmugam P., Pathak S. D. Some studies on the impact behavior of banded microalloyed steel // Engineering and Fracture Mechanics. - 1996. - Vol. 53, iss. 5. - P. 991-1005. DOI: 10.1016/0013-7944(95)00159-X.

14. Toughness deterioration in advanced high strength bainitic steels / F. G. Caballero, J. Chao, J. Cornide, C. García-Mateo, M. J. Santofimia, C. Capdevila // Materials Science and Engineering A. - 2009. - Vol. 525. - P. 87-95. - DOI: 10.1016/j.msea.2009.06.034.

15. ASTM E 1268-01. Standard Practice for Assessing the Degree of Banding or Orientation of Microstructures. - ASTM, 2001. - 29 p.

16. D'Errico F. Failures induced by abnormal banding in steels // Journal of Failure Analysis and Prevention. - 2010. - Vol. 10, iss. 5. - P. 351-360. - DOI: 10.1007/s11668-010-9374-3.

17. Rodionova I. G., Zaitsev A. I., Baklanova O. N. Effect of Carbon Steel Structural Inhomogeneity on Corrosion Resistance in Chlorine-Containing Media // Metallurgist. - 2016. - Nos. 9-10. P. 774-783. - DOI: 10.1007/s11015-016-0173-2.

18. Rivera-Diaz-del-Castillo P. E. J., Van der Zwaag S. Assuring Microstructural Homogeneity in Dual Phase and TRIP Steels // Steel Research International. - 2004. - Vol. 75, no. 11. P. 711-715. - DOI: 10.1002/srin.200405832.

19. Xu W., Rivera-Diaz-del-Castillo P. E. J., Van der Zwaag S. Ferrite/Pearlite Band Prevention in Dual Phase and TRIP Steels: Model Development // ISIJ International. - 2005. - Vol. 45, no. 3. P. 380-387. - DOI: 10.2355/isijinternational.45.380.

20. Marder A. R. Deformation characteristics of dual-phase steels // Metallurgical Transactions A. - 1982. - Vol. 13. - P. 85-92. - DOI: 10.1007/BF02642418.

21. Cai X. L., Garratt-Reed A. J., Owen W. S. The development of some dual-phase steel structures from different starting microstructures // Metallurgical Transactions A. - 1985. - Vol. 16. P. 543-557. - DOI: 10.1007/BF02814228.

22. Jatczak C. F., Girardi D. J., Rowland E. S. On banding in steel // Transactions of ASM. 1956. - Vol. 48. - P. 279-305.

23. ГОСТ 5640-68. Металлографический метод оценки микроструктуры листов и ленты. М. : Издательство стандартов, 1988. - 18 с.

24. ГОСТ 801-78. Сталь шарикоподшипниковая. Технические условия. М. : Издательство стандартов, 1979. - 45 с.

25. UNI 8449. Classification of banded structure in case hardening steels. - UNI, 1983. - 15 p.

26. Tasan C. C., Hoefnagels J. P. M., Geers M. G. D. Microstructural banding effects clarified through micrographic digital image correlation // Scripta Materialia. - 2010. - Vol. 62, iss. 11. P. 835-838. - DOI: 10.1016/j.scriptamat.2010.02.014.

27. Krebs B., Hazotte A., Germain L. Quantitative analysis of banded structures in dual-phase steels // Image Analysis and Stereology. - 2010. - Vol. 29, no. 2. - P. 85-90. DOI: $10.5566 /$ ias.v29. p. 85-90.

28. ГОСТ Р 54570-2011. Методы оценки степени полосчатости или ориентации микроструктур. - М. : Стандартинформ, 2012. - 36 с. 